\title{
Lipid-mediated Association of the Slg1 Transmembrane Domains in Yeast Plasma Membranes
}

A. Alavizargar, A. Elting, R. Wedlich-Söldner, A. Heuer 


\begin{abstract}
Clustering of transmembrane proteins underlies a multitude of fundamental biological processes at the plasma membrane such as receptor activation, lateral domain formation and mechanotransduction. The selfassociation of the respective transmembrane domains (TMD) has also been suggested to be responsible for the micron-scaled patterns seen for integral membrane proteins in the budding yeast plasma membrane (PM). However, the underlying interplay between local lipid composition and TMD identity is still not mechanistically understood. In this work we have used coarse-grained molecular dynamics (MD) simulations as well as microscopy experiments (TIRFM) to analyze the behavior of a representative helical yeast TMD (Slg1) within different lipid environments. Via the simulations we evaluated the effect of acyl chain saturation and the presence of anionic lipids head groups on the association of TMDs via simulations. Our simulations revealed that weak lipid-protein interactions significantly affect the configuration of TMD dimers and the free energy of association. Increased amounts of unsaturated phospholipids strongly reduced helix-helix interaction and the presence of phosphatidylserine (PS) lipids only slightly affected the dimer. Experimentally, the network factor, characterizing the association strength on a mesoscopic level, was measured in the presence and absence of PS lipids. Consistently with the simulations, no significant effect was observed. We also found that formation of TMD dimers in turn increased the order parameter of the surrounding lipids and induced long-range perturbations in lipid organization, shedding new light on the lipid-mediated dimerization of TMDs in complex lipid mixtures.
\end{abstract}

\title{
1 Introduction
}

The plasma membrane (PM) has to fulfill a wide range of biological functions such as selective uptake of nutrients and ions, signal transduction and cellular morphogenesis. For these diverse processes to be regulated efficiently, many resident proteins and lipids of the PM are laterally segregated into distinct functional domains. A striking example of such domains has been described for the budding yeast PM, where all integral membrane proteins as well as lipids form a patchwork of overlapping domains [1]. A systematic microscopic evaluation of yeast PM resident proteins demonstrated that domain formation depends on both the sequence of TMDs and the cellular lipid composition [2]. Based on these findings, it was suggested that collective interactions between TMDs and lipids could drive the formation of characteristic patchwork membrane [3]. A particularly powerful observable is the network factor (denoted intensity distribution in [2]) which is a measure for the heterogeneity of the arrangement of a specific PM marker. Homogeneous arrangement in the PM yields a large network factor, strong clustering a small value. Thus, on a qualitative level the network factor can be regarded as a mesoscopic observable to characterize the effective association tendency of TMDs. However, a mechanistic understanding of lateral TMD association and segregation in the yeast PM is still lacking.

One way to approach this problem is to perform MD simulations of simplified mixtures of TMDs and lipids. Most TMDs consist of single $\alpha$-helices with mostly hydrophobic or small amino acids. Many experimental techniques have been used to probe the factors involved in the association process of TMDs. 
These factors include sequence-dependent parameters of the TMDs, the surrounding lipid environment, binding of ligands to the external or internal functional groups as well as general physico-chemical properties of the membrane [4]. Concerning the former factor, many TMDs contain specific sequence motifs. Several studies have identified GxxxG motif as dimerization motif, where $\mathrm{x}$ stands for some arbitrary hydrophobic residue with a short or slightly polar side chain $[5,6]$, and allows a good steric match between the interacting surfaces of TMDs. A more general 'GxxxG-like' motif has been proposed as SmxxxSm, where 'Sm' is a small residue (Gly, Ala, Ser or Thr) [7]. It has been shown that GxxxG-like motifs can influence both the free energy of helix-helix association and the structural conformation of TMD dimers, characterized by specific crossing angles [4].

The biophysical properties of the lipid bilayer such as lipid order or hydrophobic thickness can have additional impact on the association of TMDs, the so-called non-specific effects. On the one hand, highly ordered lipids in the gel phase exclude TMDs and thereby enhance local TMD concentration and dimerization [8]. On the other hand, increasing lipid order and the transition from Ld to Lo phase by the addition of cholesterol directly increases association of TMDs $[9,10]$. In addition to its non-specific effects on lipid order and membrane thickness, cholesterol has been shown in coarse grained simulations to specifically interact with TMD stretches, including GxxxG or CRAC motifs [11], also suggested recently in by NMR experiments [12]. Specific examples include the TMDs of ErbB2 [13, 14] or Glycophorin A (GpA) [15]. In addition, the helix-helix association is clearly affected by the hydrophobic thickness of the bilayer $[16,17]$, which has also been studied computationally [18-21], or the combination of both [22]. Experimentally, increased thickness of model membranes through longer acyl chains or addition of cholesterol promotes the self-association of TMDs [8]. Other examples of the effect of non-specific proteinlipid interactions for TMD association, including both simulations experiments, are also present: dimerization of bitopic TMDs is affected by the length and degree of saturation of lipids acyl chains [15, 23, 24] and charge [25] in the surrounding lipids of the bilayer. MD simulations have also inspected the comparative effects of lipids decoupling from TMD dimers [26, 27].

While bilayer properties and composition clearly regulate the association of TMDs, the association of proteins in turn can have large scale effects on the lipid environment. MD simulations have shown that a single TMD can already induce long-range perturbations in the hydrophobic core of the bilayer, suggesting to mediate communication between TMDs, which are initially located at a distance of 2-3 nm from each other [28]. A reduction in membrane perturbation in terms of membrane thickness and order has also been observed upon helix association [29]. Local membrane thickness variations have been also observed around dimers in model membranes with different lipid compositions [24].

Despite the number of reports on selected TMD membrane systems, the impact of weak, collective forces on helix-helix association as well as the complex interplay between dimer formation and local lipid properties is still insufficiently understood.

In this work, we combine computational and experimental approaches to study TMD-association in a lipid bilayer system mimicking the yeast PM. In order to select a representative TMD for our studies, we tested localization of isolated TMDs from various single-spanning integral yeast PM proteins. Of 
those that were correctly delivered to the PM, only four contained the previously characterized GxxxG motifs the cell wall stress sensors Mid2, Mtl1 and Slg1 and the cell fusion protein Fus1. We decided to focus on the Slg1 TMD, as it exhibited a total of three GxxxG motifs and one GxxxG-like motif (Figure 1A). The first two GxxxG motifs, called glycine zipper, have been previously recognized as a driving motif for homo- and oligomerization of membrane proteins [30-32]. In addition, the central GVxxGV motif has been identified as a strong interaction motif in the GpA TMD dimer [7] (Figure 1A). Finally, the cytosolic C-terminus of the Slg1 TMD contains two positively charged residues (Figure 1A) that might affect TMD association via electrostatic interaction with anionic lipid head groups.

For the MD simulations, we use the coarse-grain MARTINI force field to allow sufficient sampling for free energy calculations. Specifically, we simulated the dimerization of two identical TMDs in an ergosterol containing bilayer environment upon variation of phospholipid composition. We specifically focused on the degree of saturation and presence or absence of anionic phosphatidylserine (PS). The chosen phospholipid and ergosterol concentrations mimic the situation in the yeast PM. TMD dimer formation is then characterized via the equilibrium ratio of left- and right-handed dimers as well as by the free energy of dimer formation. Both properties are quantified using umbrella sampling. To increase the level of information we then compare results from a large number of independent simulations of TMD dimer formation, starting from two separated TMDs. Finally, we show how the bidirectional interplay between TMDs and lipids can act as a self-regulating process to stabilize dimers. Due to the direct interaction of anionic PS with the TMD, as clearly seen in the MD simulations, surprisingly, omission of PS turned out to have no relevant impact on the free energy of TMD dimer formation. This result is consistent with high-resolution microscopy experiments, where the network factor does not depend on the PS content as well. 


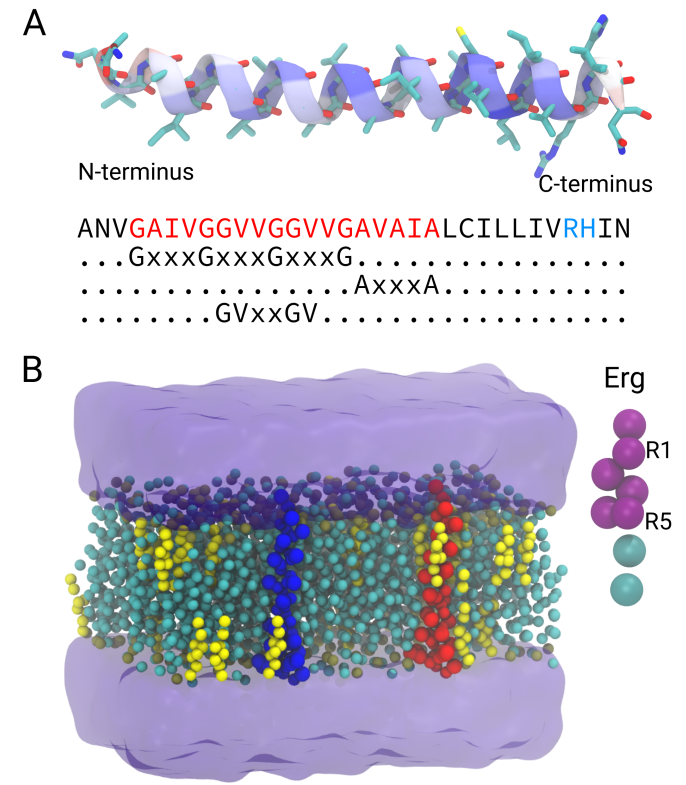

Figure 1: (A) The atomistic representation of the Slg1 TMD studied in this work, along with the associated sequence. The GxxxG and GxxxG-like motifs as well as the positively charged residues in the $\mathrm{C}$-terminus are highlighted, respectively, in red and blue. (B) The simulations system is represented with the two TMD located at a distance of $4.5 \AA$ from each other. The beads of the two TMDs and the lipids are shown in spheres (ergosterol molecules in yellow) and the water is shown in surface representation. Half of the simulation system is not shown for clarity. Ergosterol molecule is shown in MARTINI representation and the R1 and R5 (the first and the last bead of the planar part) are labeled.

\section{Methods}

\section{$2.1 \quad$ Experimental basis}

\subsubsection{Yeast strains plasmids}

The yeast strains created in this study were derived from Saccharomyces cerevisiae BY4741 MATa (Euroscarf). The Slg1TMD-plasmid was constructed using standard molecular biology techniques to express ANVGAIVGGVVGGVVGAVAIALCILLIVRHIN-GAGAGAGAGAmNeonGreen from the strong Pma1 promoter. Transformation into yeast cells was performed using the lithium acetate method. All constructs and were verified by sequencing.

Yeast strains:

\begin{tabular}{lll}
\hline Strain & Genotype & Source \\
\hline BY4741 & MATa his3 $\Delta 1$ leu2 $\Delta 0$ met152 $\Delta 0$ ura32 $\Delta 0$ & Euroscarf \\
RWS1616 & BY4741 cho1::natNT2 & Spira, 2012 \\
RWS5149 & BY4741 RWC1583 & This study \\
RWS5150 & BY4741 cho1::natNT2 RWC1583 & This study \\
\hline
\end{tabular}


Plasmids:

\begin{tabular}{lcccc}
\hline Plasmid & Backbone & Description & Marker & Source \\
\hline RWC1583 & pRS316 & pPma1-Slg1-TMD[260-291]-5xGA-mNeonGreen & URA3 & This study \\
\hline
\end{tabular}

\subsubsection{Yeast cell culture}

The plasmid-containing strains were grown overnight in synthetic complete media without uracil and $2 \%$ glucose $+1 \mathrm{mM}$ ethanolamine (SCD -Ura + etn) media at $30^{\circ} \mathrm{C}$ with shaking, washed $3 \mathrm{x}$ with $\mathrm{H} 2 \mathrm{O}$, diluted to $\mathrm{OD} 600=0.1$ in $\mathrm{SCD}$-Ura + etn, further grown for $2.5 \mathrm{~h}$ at $30^{\circ} \mathrm{C}$ and imaged in mid-logarithmic phase.

\subsubsection{Microscopy and imaging}

Epifluorescence and total internal reflection fluorescence microscopy (TIRFM) were performed on a fully automated iMIC stand (FEI/Till Photonics) with an Olympus $100 \times 1.45 \mathrm{NA}$ objective. DPSS lasers $(75 \mathrm{~mW})$ at $491 \mathrm{~nm}$ (Coherent Sapphire) and $561 \mathrm{~nm}$ (Cobolt Jive) were selected through an acousto-optical tunable filter. Images were collected with an Andor iXON DU-897 EMCCD camera controlled by the Live Acquisition (Till Photonics) software. For imaging, coverslips (Knittel Glass No. 1) were cleaned by sonication in absolute ethanol (Sigma), $>99.5 \%$ acetone (Sigma), $1 \mathrm{M} \mathrm{NaOH}$ (Roth), ddH2O and finally stored in absolute ethanol. To immobilize cells, coverslips were coated with $1 \mathrm{mg} / \mathrm{ml}$ concanavalin A (Sigma).

\subsubsection{Image processing and analyses}

Microscopy of the yeast PM was performed as described previously [33]. Images were processed using Fiji and MATLAB (MathWorks Inc., Natick, MA). Images were contrast-adjusted and scaled for presentation purposes only. All TIRFM images were deconvolved using the LucyRichardson algorithm in MATLAB using 12 iterations. PSF were calculated from images of $100 \mathrm{~nm}$ tetra spec beads images. The network factor describes the normalized GFP intensity distribution at the PM and was calculated from TIRFM images using a custom-written MATLAB script an. All images shown were scaled 3x using Fiji.

\subsubsection{Lipidome analysis}

The yeast strains created in this study were derived from Saccharomyces cerevisiae BY4741 MATa (Euroscarf). The Slg1TMD-plasmid was constructed using standard molecular biology techniques to express ANVGAIVGGVVGGVVGAVAIALCILLIVRHIN-GAGAGAGAGAmNeonGreen from the strong Pma1 promoter. Transformation into yeast cells was performed using the lithium acetate method. All constructs and were verified by sequencing. 


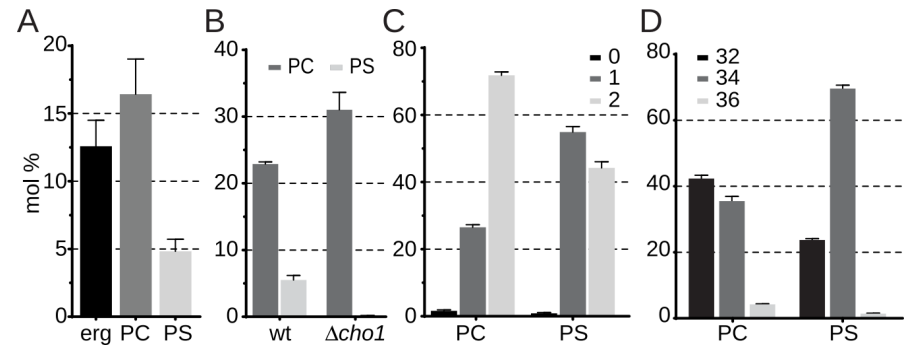

Figure 2: Lipid composition. Lipid composition of yeast membranes. (A) The relative amounts of ergosterol, PC and PS in control cells at $30^{\circ} \mathrm{C}$, (B) The depletion of PS in the $\Delta$ cho1 mutant, (C) the acyl chain saturation for PC and PS and (D) the length of acyl chains for PC and PS are represented.

\subsubsection{Determination of lipid composition}

Lipid-extractions, mass-spectrometric analyses, quantification, and data processing were performed by Lipotype GmbH (Dresden, Germany). To generate samples, yeast cultures were incubated overnight in SCD $+1 \mathrm{mM}$ choline at $30 \mathrm{C}$ with shaking, diluted to an OD600 of 0.1 in SCD $+1 \mathrm{mM}$ choline and further grown at 30C to mid-logarithmic phase. $20 \mathrm{OD}$ were harvested for extract preparation and shipped to Lipotype. Lipidome-results in this study were normalized to total lipids of selected categories and compared using Excel. To determine the typical lipid composition of yeast membranes in our culture conditions, we performed lipidomic analysis. Considering the reduced lipid complexity required to achieve sufficiently large MD simulation times, we focused on three major lipid constituents of the yeast PM: ergosterol (the main yeast sterol), the apolar phosphatidylcholine (PC) and the anionic PS that make up 13, 16 and $5 \%$ of all lipids in yeast membranes, respectively (Figure 2A). Values in Figure 2A and $\mathrm{B}$ do not add up to $100 \%$ as only three of the 9 lipid classes detected are shown. Note that both ergosterol and PS are strongly concentrated in the yeast PM $[34,35]$ and PS is highly restricted to the inner bilayer leaflet [34, 36, 37]. The effective concentrations of ergosterol and PS in the PM are therefore far higher $(>20 \%)$ than the overall average values [36] measured. We also used the $\Delta$ cho1 mutant that is not able to synthesize PS anymore (Figure $2 \mathrm{~B}$ ). Note that $\mathrm{PC}$ and PS in yeast slightly differ in their degree of saturation (Figure $2 \mathrm{C}$ ) and acyl chain lengths (Figure 2D), with most PC containing two unsaturated and shorter acyl chains (C16:1/C16:1, C16:1/C18:1) and PS featuring more mixed chains (C16:0/C18:1, C16:1/C18:1, C16:1/C18:0). Also note that budding yeast only expresses a single desaturase enzyme so that acyl chains typically contain no more than a single double bond.

\subsection{Simulation basis}

\subsubsection{Choice of model}

The initial atomistic structure of the Slg1 TMD was prepared via SWISSMODEL homology modeling webserver [38]. The structure was replicated in a way that the two monomers are located at the distance of $4.5 \AA$ from each other. The two monomers were inserted in membranes with different composi- 
tions using Martini maker as implemented in web-based CHARMM-GUI membrane builder [39]. Accordingly, the systems were solvated by water molecules and ions were added to neutralize the systems. The final systems consist of two TMD domains and around 400 lipid molecules (Figure 1B). Five different systems with different compositions were prepared. All the systems contain $20 \%$ ergosterol. One system with POPC (16:0,18:1) and ergosterol (POPC-Erg) was additionally created. Two systems out of five have the most similar lipid composition as the implemented experiments (previous section), one without PS lipids (DOPC-POPC-Erg) and the other with PS lipids (DOPC-POPC-Erg-PS). The PS lipids are present only in the lower leaflet of the membrane and their amount is one sixth of the non-PS lipids present in the lower leaflet, excluding ergosterol. It should be noted that due to the coarse-grain structure of the lipids in MARTINI, not all the lipid types in the actual experiment could be reproduced, and therefore, the lipid composition is only an approximation. Two extra systems were also constructed similar to the experimental composition with the exception that DOPC $(18: 1,18: 1)$ is replaced by a more unsaturated DIPC $(18: 2,18: 2)$ lipid (DIPC-POPC-Erg, DIPC-POPC-Erg-PS) in order to probe the effect of saturation status. The percentage of each lipid type in all membrane systems is represented in Table 1.

Table 1: The percentage of the lipid components for each system, averaged over the whole membrane. Note that the PS lipids are present only in the lower leaflet.

\begin{tabular}{lccccccc}
\hline System & POPC & DOPC & DIPC & POPS & DOPS & DIPS & Erg \\
\hline POPC-Erg & 80 & 0 & 0 & 0 & 0 & 0 & 20 \\
DOPC-POPC-Erg & 26.7 & 53.3 & 0 & 0 & 0 & 0 & 20 \\
DOPC-POPC-Erg-PS & 22.9 & 45.7 & 0 & 3.8 & 7.6 & 0 & 20 \\
DIPC-POPC-Erg & 26.7 & 0 & 53.3 & 0 & 0 & 0 & 20 \\
DIPC-POPC-Erg-PS & 22.9 & 0 & 45.7 & 3.8 & 0 & 7.6 & 20 \\
\hline
\end{tabular}

\subsubsection{Simulations of TMD association}

To study the association of the Slg1 TMDs in different bilayer systems, coarsegrain molecular dynamics (MD) simulations were performed. To describe the interactions between the peptides, lipids and solvent molecules, the version 2.2 of MARTINI force field was used [40-42]. In MARTINI force field, on average 4 atoms along the associated hydrogens are represented by one interaction site (bead). The MD simulations were performed using the version 2019 of GROMACS [43, 44]. The constant temperature was controlled by coupling the system to a Berendsen thermostat [45] at $310 \mathrm{~K}$. The pressure was controlled by coupling the system to a barostat (coupling time of 0.1 ps and compressibility of $3 \times 10^{-4}$ bar $^{-1}$ ) at 1 bar, using semi-isotropic coupling scheme [45]. For the production simulations the constant pressure was controlled using the Parinello-Rahman barostat [46]. The non-bonded interactions were treated using a switch function from 0.0 to $1.1 \mathrm{~nm}$ for the electrostatic, and from 0.9 to $1.1 \mathrm{~nm}$ for Lennard Jones interactions. The equilibration procedure was performed according to the input files provided by the CHARMM-GUI webserver. For each system, 29 independent samples were simulated and the simulation 
time for each sample is $5 \mu$ s performed in the NPT ensemble with the time step of $20 \mathrm{fs}$. In the rest of the paper, we refer to these simulations as 'ensemble' simulations.

All the simulations data have been analyzed using the python routines incorporating the MDAnalysis package [47, 48] and GROMACS tools. The VMD software was used to visualize the trajectories [49].

\subsubsection{Crossing angle and tilt angle of TMDs}

The crossing angle was calculated according to ref. [50]. Accordingly, the crossing angle is the dihedral angle constructed by the beginning of each helix and the two points on the two helices, which are at the closest distance from each other. The beginning of the helix is considered as the center of mass (COM) distance of the backbone of the last three residues on the $\mathrm{N}$-terminus side of the helices. The right-handed $(\mathrm{RH})$ and left-handed $(\mathrm{LH})$ configuration correspond, respectively, to negative and positive crossing angles.

The average tilt angle of the helices was calculated as the angle between the vector connecting the beginning and end of each helix and the membrane normal (z-axis). The beginning and end of the helix was considered as the COM of the first three and three last three residues of the helix, considering the backbone beads.

\subsubsection{Potential of mean force}

In order to estimate the stability of the dimer in different membranes, we calculated the potential of mean force (PMF) profile for the TMDs dissociation. For this purpose, we used umbrella sampling [51]. The reaction coordinate was chosen as the COM distance of the two TMDs, considering the backbone beads. The starting structures for each window was prepared as follows: first, starting from a frame in which a $\mathrm{RH}$ or $\mathrm{LH}$ dimer is formed, and then one TMD was pulled using the pull scheme of GROMACS with the rate of $0.1 \mathrm{~nm}$ per ns, while the other TMD is restrained with a force constant of $1000 \mathrm{~kJ} \mathrm{~mol}^{-1} \mathrm{~nm}^{-2}$. In each window, the umbrella potential was applied so as the COM distance is restrained with the force constant of $1000 \mathrm{~kJ} \mathrm{~mol}^{-1} \mathrm{~nm}^{-2}$. 29 windows were run with the window size of $0.1 \mathrm{~nm}$. Each window was first equilibrated for 50 ns, followed by 8 to $10 \mu$ s production simulations. The WHAM method was employed to unbias the umbrella potentials and to combine all the windows [52].

\subsubsection{Order parameter of phospholipids and tilt angle of ergosterol}

The order parameter measures how the lipid chains are oriented with respect to the membrane normal and quantifies the degree of their orientational order. According to ref. [53] the order of lipid chains can be quantified using molecular order parameter $\left(S_{m o l}\right)$

$$
S_{m o l}=\frac{1}{2}\left\langle 3 \cos ^{2} \Theta_{n}-1\right\rangle
$$

where $\Theta_{n}$ is the angle between the vector constructed by $\mathrm{n}^{\text {th }}$ segment of the hydrocarbon chain, i.e., $C_{n-1}$ and $C_{n+1}$, connecting the $n-1$ and $n+1$ carbon atoms, and the membrane normal (z-axis). For the coarse-grain lipids, however, 
the vector connecting the two consecutive beads is considered, i.e., $C_{n-1}$ and $C_{n}$. Subsequently, this quantity is calculated for all the beads of the two chains separately. The angular brackets represent the time and ensemble average.

The tilt angle of ergosterol was analyzed considering the planar part and was taken as the angle between the vector connecting R1 and R5 beads and the membrane normal (z-axis) (Figure 1B).

\section{Simulation results}

To systematically study the interactions between a yeast TMD and the lipid bilayer, we simulated the dimerization/association of two Slg1 TMD molecules (Figure 1A), using coarse-grain MD simulations (Figure 1B), and explored the effects of lipid environment on the TMD association - and vice versa.

\subsection{Dimerization properties induced by membrane envi- ronment}

\subsubsection{Association of TMDs}

The self-association of the TMDs was studied. In the initial structure, the two TMDs were separated with the distance of $4.5 \mathrm{~nm}$ in order to eliminate the initial interactions between monomers (Figure 1B). During the $5 \mu$ s simulation for each sample of ensemble simulations (see Methods section), on average the dimer is formed in $92.8 \%$ of all the independent simulation samples, considering all systems; see Figure S1 for a representative example.

Before the dimer is formed, a particular pathway is followed. Here, the tilt angle can describe how the two TMDs approach each other until a compact dimer is formed (Figure 3). Indeed, the average tilt angle of the TMDs is reduced upon dimerization and a systematic distance dependence is observed: as the two TMDs approach each other, the tilt angle is reduced. For $12<\mathrm{COM}<\sim 20 \AA$, it continuously decreases as the two TMDs come closer to each other. This region may be interpreted as a pre-dimerized state, in which the two TMDs interact mainly through the C-terminus residues, in a way that a "V"-shaped structure is formed. This is also reflected in the average number of contacts between the corresponding similar residues on the two TMDs for $10<\mathrm{COM}<20$ $\AA$ (Figure S2). For COM $<8 \AA$, the tilt angle is the lowest and is nearly constant (dimer). Here we choose $10 \AA$ to define the transition between the dimer state and pre-dimerized state.

The tilt angle of TMDs strongly depends on the chosen lipids. In DOPC systems the tilt angle is significantly higher than in DIPC systems, whereas the presence of the PS lipids has a negligible impact.

The configuration of the two TMDs with respect to each other and the final dimer structure can be characterized by a crossing angle (see simulation basis). Accordingly, there are two possible configurations for both the pre-dimerized state and final dimer: right-handed $(\mathrm{RH})$ and left-handed $(\mathrm{LH})$ crossing angles. At large COM distances, where the TMDs are far from each other, the crossing angle is around zero due to symmetry reasons (Figure 4A). As the two TMDs approach each other, the average crossing angle increases and, interestingly, it displays a similar behavior for all the systems in the pre-dimerized state 


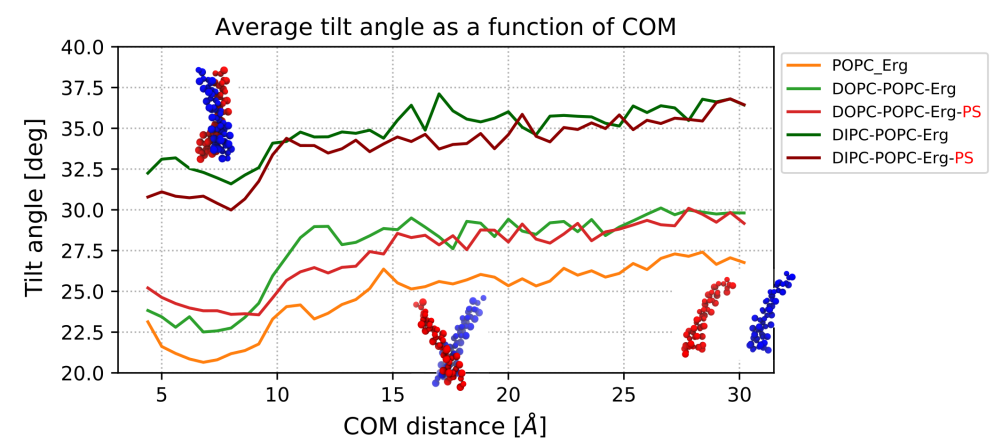

Figure 3: The average tilt angle of the two TMDs as a function of the COM distance between the two TMDs. The snapshots of the two TMDs are also shown for different COM distances.

$(10<\mathrm{COM}<20 \AA)$ since it is mainly positive. This means that the LH configurations are the main intermediate structures, which can subsequently lead to either RH or LH final dimers. This positive average value is a consequence of a broad distribution of the positive values (reaching values up to $75^{\circ}$ ) as compared to the distribution of negative values (Figure 4B). Generally speaking, this suggests that there is a universal path towards the formation of the binding state. The binding state itself displays a much stronger dependence to the lipid composition, discussed as follows.

Upon dimerization in ensemble simulations, the crossing angle values are decreased and the form of the distribution depends on the system (Figure 4C). The complex mixtures with DIPC lipids represent mainly $\mathrm{RH}$ configurations, whereas POPC-Erg as well as complex mixtures with DOPC lipids form both RH and LH dimers, with nearly similar probabilities (Table 2). Interestingly, LH structures are less well-defined than RH structures, as reflected by their broader distribution of crossing angles: the average crossing angle for $\mathrm{RH}$ configurations is $\sim 25^{\circ}$, whereas $\mathrm{LH}$ configurations exhibit either similar or higher values of crossing angle when compared to RH ones. In LH configurations, L26 and H30 residues, which have relatively longer and bulkier side chains, face each other, while in RH configurations they are relatively far from one another (Figure 4D). As noted above, these residues have a significant role also in pre-dimerization via the C-terminus and their interaction initializes the dimerization process (Figure S2). Therefore, the universal pathway in the pre-dimerized state, as discussed above, is expected since the attachment of these residues induces LH configurations. 


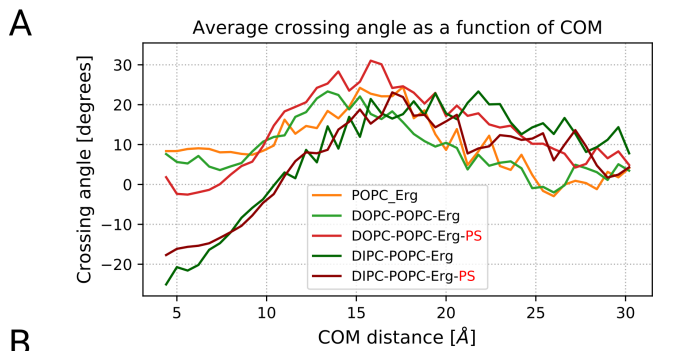

B

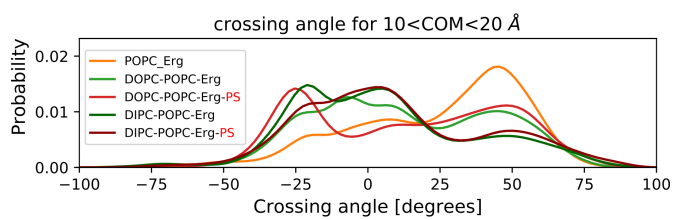

C

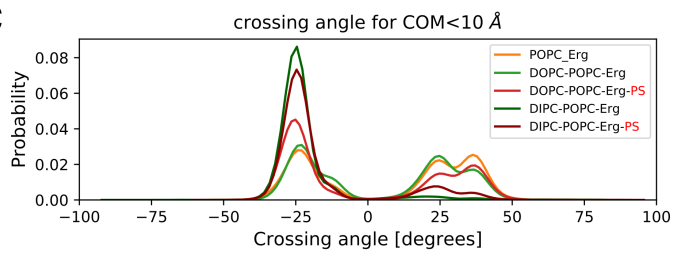

D
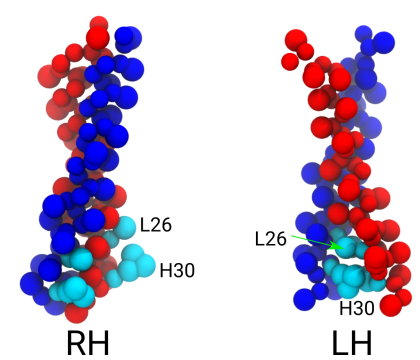

$\mathrm{LH}$

Figure 4: (A) The average crossing angle of the two TMDs as a function of COM distance between the two TMDs is shown. (B) The distribution of crossing angle for $0<\mathrm{COM}<10 \AA$ and $(\mathrm{C})$ for $10<\mathrm{COM}<20 \AA$ is shown. (D) The $\mathrm{RH}$ and $\mathrm{LH}$ configurations of the dimer are shown. The L26 and H30 residues are depicted in cyan.

Table 2: The percentage of RH and LH configurations collected from all the association samples in ensemble simulations in which the dimer is formed as well as the ones extracted from the associated $\Delta \mathrm{G}$ values is shown.

\begin{tabular}{lcc}
\hline System & \%RH in ensemble sim. & \%RH estimated using $\Delta \mathrm{G}$ \\
\hline POPC-Erg & $48 \pm 9$ & 71 \\
DOPC-POPC-Erg & $54 \pm 9$ & 67 \\
DOPC-POPC-Erg-PS & $56 \pm 9$ & 30 \\
DIPC-POPC-Erg & $86 \pm 6$ & - \\
DIPC-POPC-Erg-PS & $79 \pm 7$ & - \\
\hline
\end{tabular}

\subsubsection{Residue contacts}

Next we looked at the TMDs interfaces in the dimer state in ensemble simulations, specifically for RH and LH configurations. For this purpose, we cal- 

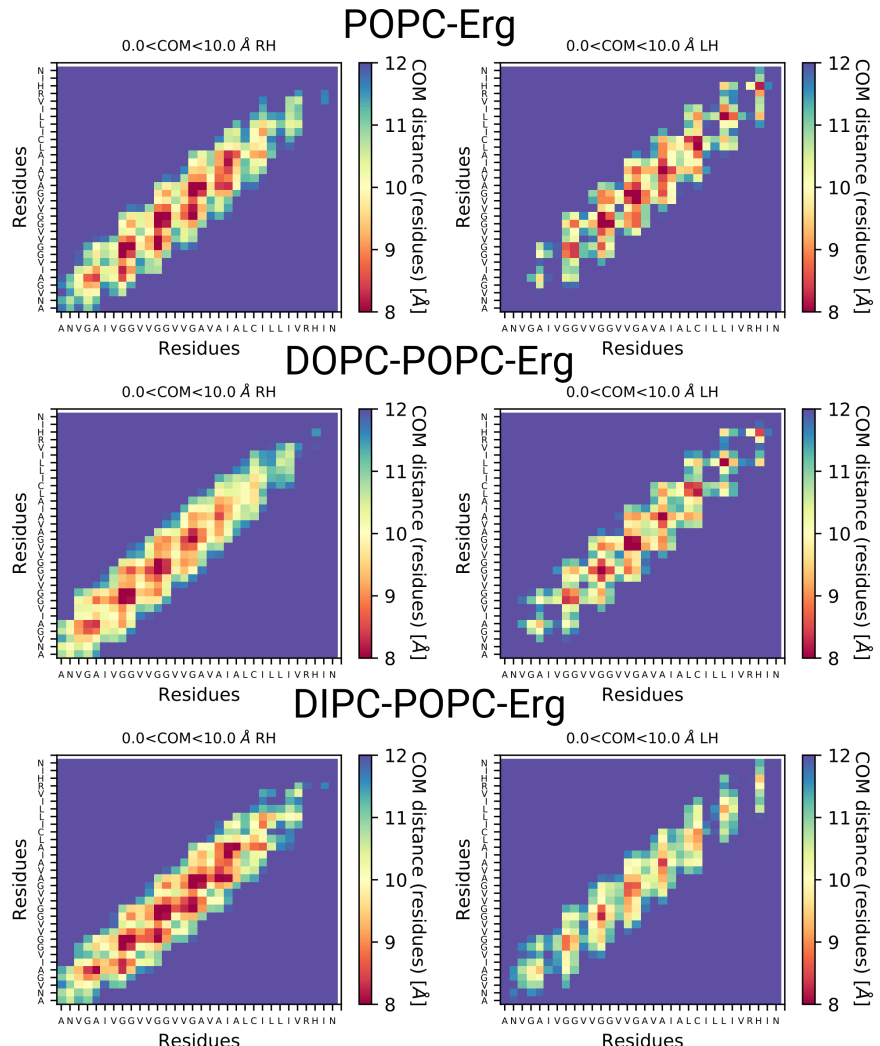

Figure 5: The contact maps of the TMD residues in different membrane systems for RH (right) and LH (left) configurations is shown. The COM distance between each residue pair is considered as a contact.

culated the contact map of the TMDs residues for $0<\mathrm{COM}<10 \AA$ (Figure 5). For POPC-Erg and DOPC systems, the number of residue contacts for RH and LH state is rather similar, which is in line with the similar probabilities of RH and LH configurations (Table 2). For DIPC systems, however, we observed a significant higher number of residue contacts for $\mathrm{RH}$ and the opposite for $\mathrm{LH}$ configurations (Figure 5). This is in agreement with the dominating probability of $\mathrm{RH}$ configurations in these systems. If we count the number of contacts for the range where the COM distance between each similar residue pair on the two TMD is between 0 and $9 \AA$, this number, for instance, for DOPC systems is 29 and 24, for RH and LH configurations, respectively, whereas the corresponding numbers for DIPC systems are 52 and 8 . This shows the similarity of the number of contacts for $\mathrm{RH}$ and $\mathrm{LH}$ configurations in DOPC system and a considerable difference in DIPC systems.

Furthermore, considering different parts of the dimer, in DOPC systems, a considerable binding is taking place also via C-terminus residues, which is mainly due to a significant number of LH configuration, as discussed earlier. In DIPC systems, however, the binding is established mainly via the N-terminus and central residues of the TMDs and the role of C-terminus residues is markedly 
lower, even in LH configurations. This is probably due to the higher tilt of the TMDs in the DIPC systems and the dominating number of RH configurations.

\subsubsection{Energetics of dimerization}

In order to estimate the stability of the dimer in different lipid environments, we quantified the free energy of dissociation, i.e., the PMF as a function of the COM distance of the two TMDs via umbrella sampling (see Methods). The shape of the free energy profiles represents a global minimum and the position of the minimum is around $7 \AA$ (Figure $6 \mathrm{~A}$ ) for all systems. As it was observed earlier, POPC-Erg and DOPC systems ended up in both $\mathrm{RH}$ and $\mathrm{LH}$ configurations with nearly similar probabilities. Therefore, for these systems we performed the PMF calculations both for RH and LH configurations. For these free energy calculations, the crossing angle values remain either $\mathrm{RH}$ or $\mathrm{LH}$ and do not switch from one to another (Figure 6B). Interestingly, a similar behavior is observed in the dimerization pathway as the ensemble simulations: irrespective of the initial configuration, the crossing angle is mainly LH in the pre-dimerized state (Figure 6C). Also interestingly, LH configurations represent weaker helix-helix interactions as compared to the RH ones in POPC-Erg and DOPC systems without PS lipids (Figure 6A,D). The opposite behavior is observed in DOPC complex mixtures with PS lipids. This might be due to the fact that in the LH configurations, the charged residues are facing each other (Figure ??D) and are hardly accessible by the PS lipids. These differences are, however, not dramatic. Considering these two different states, i.e., RH and LH, we can estimate the total free energy of dimerization: $\mathrm{e}^{-\frac{\Delta G_{\text {total }}}{R T}}=\mathrm{e}^{-\frac{\Delta G_{R H}}{R T}}+\mathrm{e}^{-\frac{\Delta G_{L H}}{R T}}$, where $\mathrm{R}$ is the gas constant and $\mathrm{T}$ is the room temperature. Accordingly, stronger helixhelix interaction is predicted for the POPC-Erg and DOPC systems (Figure 6D). Subsequently, we estimated the population of $\mathrm{RH}$ and $\mathrm{LH}$ configurations based on the free energy profiles. It is possible to obtain these populations using the corresponding $\Delta \mathrm{G}$ values (Table 2 ). Alternatively, a more precise way would be to calculate the integral of the corresponding free energy profiles over the entire reaction coordinate and from that to extract the probabilities. However, this integration over the phase space hardly changed the compositions. The probabilities for $\mathrm{RH}$ and $\mathrm{LH}$ configurations obtained from the free energy of dimerization are different from the ensemble simulations. For POPC-Erg and DOPC systems without PS lipids, the free energy predict higher probabilities for $\mathrm{RH}$ configurations when compared to the ensemble simulations, especially for POPC-Erg system (Table 2). The POPC-Erg system showed even higher probabilities for LH configurations. The results are also opposite for DOPC systems with PS lipids and the free energy predicts a considerably lower number of LH configurations as compared to the ensemble simulations (Table 2). Strictly speaking, since the the ensemble simulations are not in equilibrium and there are no transitions between pre-dimerized and dimerized states, the probabilities of the $\mathrm{RH}$ and $\mathrm{LH}$ conformers might not agree with the ones estimated from free energy calculations, which are indeed in equilibrium. This likely depends on the nature of the energetic barrier between these states and may explain why for POPC-Erg system, $\Delta \mathrm{G}$ values suggest a majority of $\mathrm{RH}$ configurations, and for DOPC systems with PS, the majority of LH configurations, in contrast to the result of the ensemble simulations.

The final free energies of dimerization for all systems show that the POPC- 
A

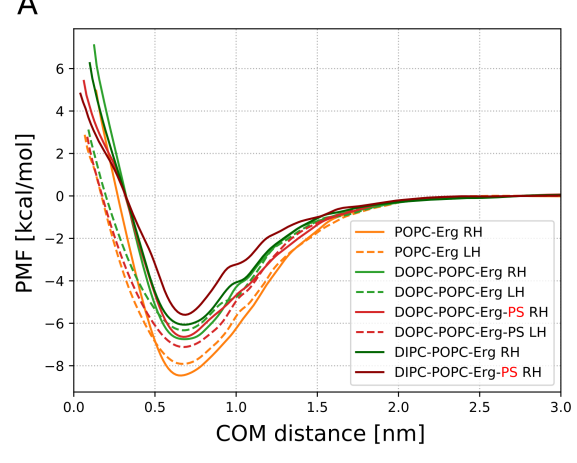

D
$\mathrm{B}$
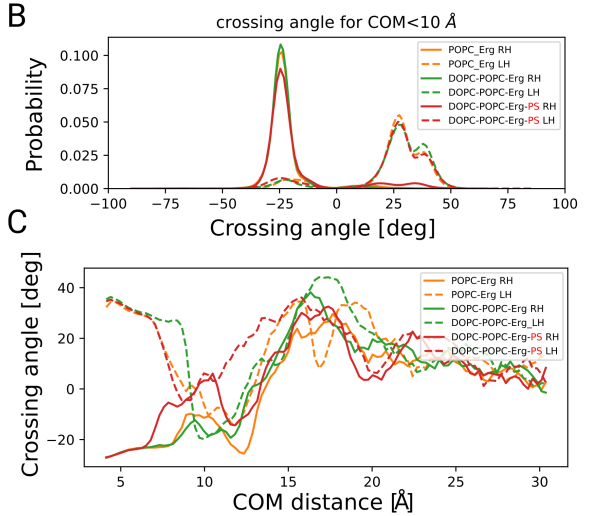

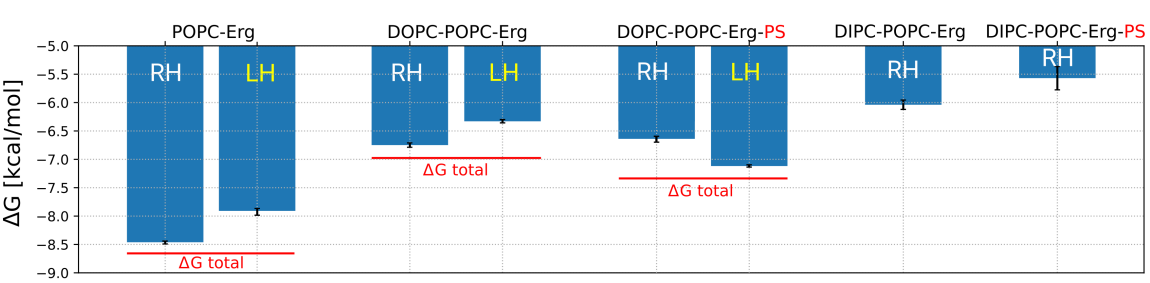

Figure 6: (A) The free energy profiles of the dimerization for all systems for RH starting structure is represented. For POPC-Erg and DOPC complex mixtures, the corresponding profiles for LH initial structure is also represented. (B) The distribution of the crossing angle and $(\mathrm{C})$ the variation of crossing angle as a function of COM distance between the two TMDs in the US simulations are shown. (D) The corresponding $\Delta \mathrm{G}$ values, i.e., the minimum of the free energy profiles in $(\mathrm{A})$ are depicted.

Erg system represents the strongest helix-helix interaction, whereas the DIPC systems display the weakest interactions (Figure 6D). Therefore, in general, the larger the number of unsaturated bonds of the surrounding phospholipids (PLs), the weaker the helix-helix interaction (DIPC $<$ DOPC $<$ POPC). Furthermore, the presence of PS lipids slightly destabilizes the dimer in DIPC complex mixtures, whereas they have the opposite effect, i.e., they stabilize the dimer in DOPC systems.

\subsection{Dimerization and relation to membrane properties}

In this section, we inspect some general properties of the studied membranes. First, we see how the thickness is changed and whether it can be related to the tilt angle of TMDs and the energy of dimerization. We also intend to probe the details of interaction of each lipid type with the TMD, and finally, how the formation of the dimer can affect the properties of the membrane, specifically the order parameter of lipids and the orientation of ergosterol molecules.

\subsubsection{Membrane thickness}

Different lipid molecules have different chain length and saturation state, and therefore, the thickness of the membrane varies in various lipid environments. On the other hand, the TMDs themselves have a specific hydrophobic length, 


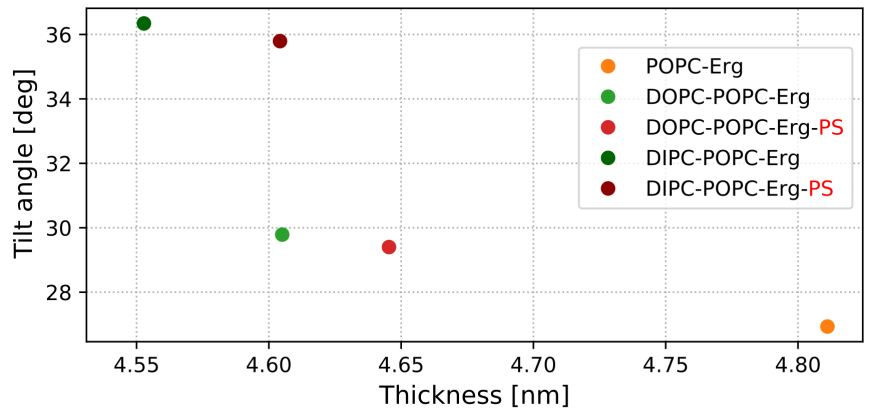

Figure 7: Average tilt angle of TMDs as a function of membrane thickness.

and thus, the TMDs and membrane naturally adjust themselves to each other $[22,54,55]$. This adjustment requires that the TMDs are tilted if their hydrophobic length is higher than the membrane hydrophobic thickness, which is the case for all the studied membranes in this work. The POPC-Erg system displays the highest thickness and the complex mixtures have nearly similar thickness values (Figure 7). The addition of PS lipids in both complex mixtures only slightly increases the thickness. The thickness is, however, weakly correlated with the TMD tilt angle. For instance, the DIPC systems display a significantly higher tilt angle although the thickness is only slightly lower than the DOPC systems. This means that thickness is not the only criterion to determine the tilt angle but other aspects of the specific interaction of the protein and the membrane are also involved. The thickness is also slightly correlated with the free energy of association: the membrane with highest thickness, i.e., POPC-Erg represents the strongest helix-helix interaction (Figure 6D).

\subsubsection{Radial distribution function}

In order to probe the lipids packing around the dimer in complex mixtures in the ensemble simulations, we quantified the radial distribution function (RDF) of the COM of the two TMDs and the head group of each lipid type in the membrane (Figure 8). In all systems, the RDF profiles show very strong interaction of ergosterol with the TMD. For the systems containing PS lipids, PS lipids represent a (weak) attractive interaction with the TMDs and approach the dimer quite closely, but not as close as ergosterol. There is a weak reduction of non-PS lipids when PS is added, which is naturally due to the fact that PS lipids take some space. The weak destabilization upon the addition of PS lipids, observed from the thermodynamics $(\Delta G)$, is reflected by the presence of weak interactions with these components. Interestingly, POPC displays an attractive interaction (i.e., $\mathrm{g}(\mathrm{r})>1$ ) only for DIPC but not for DOPC. Comparing the two complex mixtures, protein-lipid coupling already starts for distances $<3 \mathrm{~nm}$ for DIPC and $<1.7 \mathrm{~nm}$ for DOPC. This might be a consequence of the more disorderd nature of DIPC lipids with a correspondingly larger interaction range. 
A
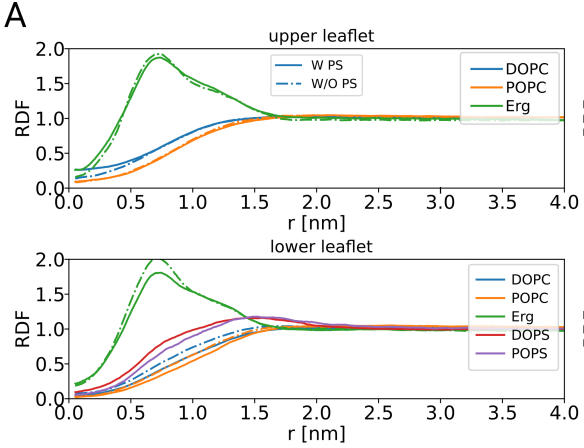

C
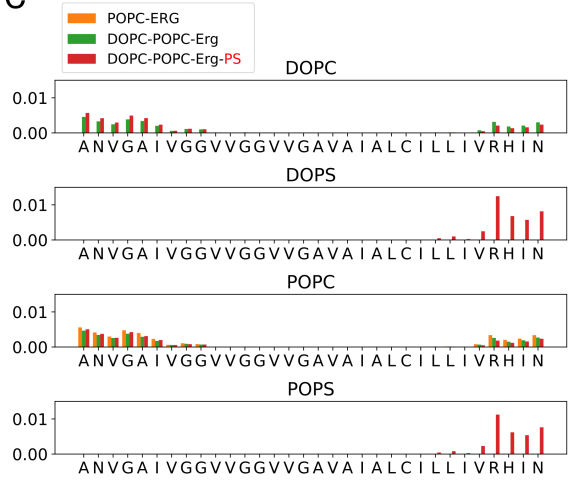

Erg

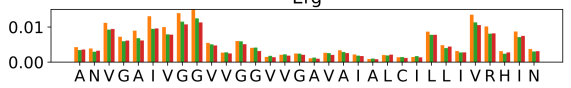

B
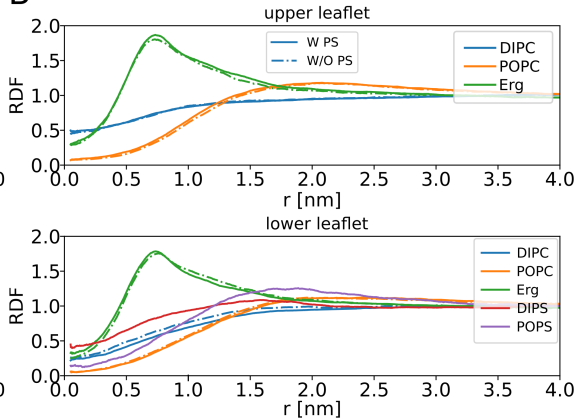

D
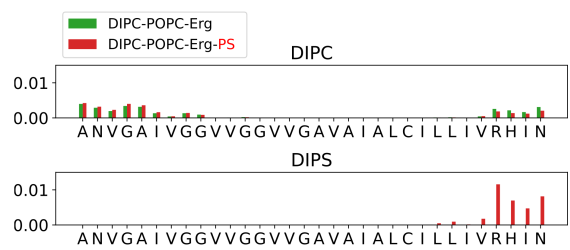

POPC

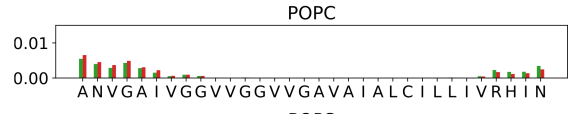

POPS

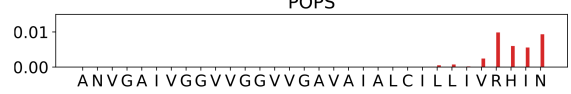

Erg

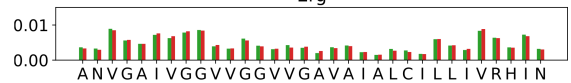

Figure 8: (A) Radial distribution function of the COM of the two TMDs and the head groups of the lipids is shown for DOPC and (B) DIPC complex mixtures, considering the configurations in which the dimer is formed. (C) The average number of contacts of the lipids head groups with the residues of the TMDs divided by the number of lipids for each type, calculated for the configurations in the dimer state for the POPC-Erg and DOPC and (D) DIPC systems is represented. A contact between each lipid type and a TMD residue is considered to be formed when the distance between the lipid head group and the COM of the considered residue is less than $6 \AA$. The number of contacts for the two TMDs were subsequently added up.

\subsubsection{Lipid-TMD contacts}

The RDF profiles of the TMDs and lipids, discussed in the previous section, provide a macroscopic picture of the lipid-protein interaction and does not give a detailed description of the interaction of the lipids with the TMD residues. Therefore, here we have analyzed the contacts of each lipid molecule with all the TMD residues in the dimer state for the POPC-Erg and the complex mixtures in ensemble simulations (Figure 8C,D). In agreement with RDF profiles, ergosterol interacts significantly with the TMDs, both in the intracellular and the extracellular side, especially with the GxxxG patterns. Interestingly, the interaction of ergosterol with the TMDs in DIPC systems is considerably different, representing significant interaction with the amino acids in the middle of the protein (Figure 8D). This might be due to the higher tilt angle of ergosterol in these systems, especially in the proximity of dimer (Figure S3). Moreover, 
the contact of ergosterol with the TMDs is not noticeably changed by the presence of PS lipids. PS lipids interact significantly with the dimer, mainly with R29, and mostly take the position of DOPC, DIPC and POPC, reflected also in the RDF profile (Figure 8C,D). Moreover, in RDF profiles, POPC displayed an attractive interaction (i.e., $\mathrm{g}(\mathrm{r})>1$ ) only for DIPC but not for DOPC, which is reflected here by the increased size of the bars. However, this also implies that the absolute values of the number of contacts are somewhat difficult to interpret since there are also contacts although the dimer is exclusively repulsive, i.e., $\mathrm{g}(\mathrm{r})<1$ for all $\mathrm{r}$ values.

\subsubsection{Order parameter of phospholipids}

So far, we have observed how the membrane environment affects the proteinprotein and protein-lipid interactions. In this section, we want to explore how the formation of the dimer can affect the membrane properties in ensemble simulations. For this purpose, we have quantified the average order parameter of the PLs, which is extensively used to describe the structural properties of the lipid bilayers. Accordingly, we estimated the order parameter both for the monomer and the dimer state (Figure 9). The average lipid order parameter hardly depends on the distance to the protein if the protein is not dimerized (Figure 9A). This means that a monomer does not have an impact on the average behavior of the acyl chains. In striking contrast, there is a much stronger perturbation of the order parameter induced by the formation of dimer (Figure 9B). Indeed, starting from large distances, the order parameter first increases until $\sim 1 \mathrm{~nm}$ (ordering effect of the dimer). For smaller distances, where all PLs experience a repulsive force, the order parameter is decreased. The increase of order parameter at intermediate distances is systematically higher if the absolute value of the order parameter is higher, i.e., for more saturated systems (Figure 9B). 

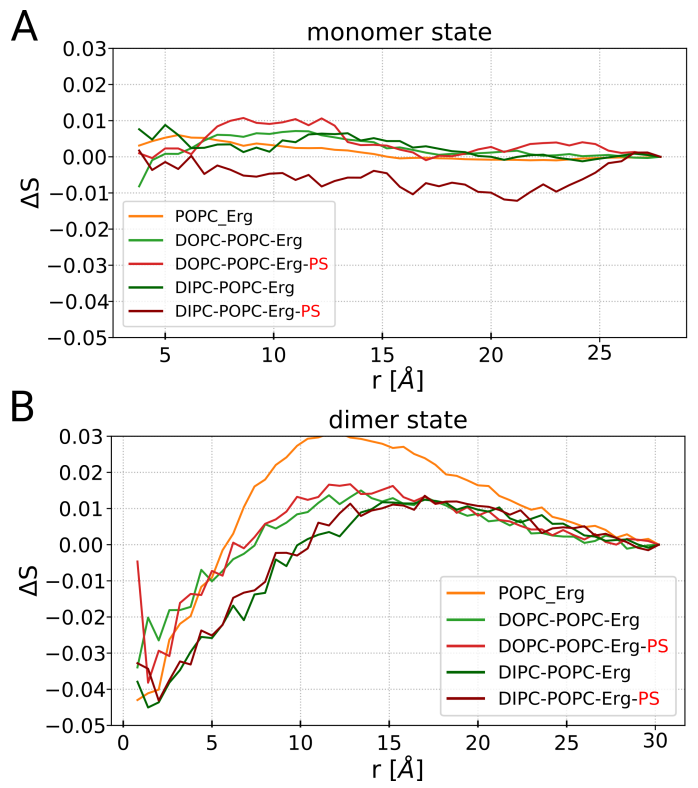

Figure 9: (A) The variation of order parameter of the PLs in monomer and (B) dimer state with respect to the order parameter values at large distances for the complex mixtures. In order to better visualize the effect of dimer on the membrane order, we superimposed the graphs for all systems with respect to the order parameter at large distances. The values of the order parameter at large distances for different systems are $0.39,0.32,0.33,0.23,0.24$ for the respective systems listed in the legend.

\section{Experimental results}

As discussed above, one way to connect atomistic simulations and mesoscopic microscopy experiments is to check the impact of a systematic variation of the lipid properties. As shown in the Methods section, we were able to experimentally control the presence of anionic PS lipids in the yeast PM and thus can check the relevance of anionic lipids.

When we tested the localization of GFP fusions to membrane segments from known single spanning yeast PM proteins, we observed correct delivery of the Slg1 ${ }^{\text {TMD }}$ fusion protein to the PM. We then examined localization of Slg1 $1^{\text {TMD }}$ mNeonGreen in more detail using the combination of TIRFM and deconvolution that we previously introduced. Slg1 $1^{\mathrm{TMD}}$ distributed in a typical network pattern (Figure 10A) that we had observed for most integral PM proteins of budding yeast $([2])$.

The key observable, characterizing the spatial distribution of TMDs in the PM is the network factor, as introduced in [2]. It turns out that in wild-type cells (in the presence of PS) the network factor of Slg1 $1^{\mathrm{TMD}}$ is around 0.27 (Figure 10B). This is in the upper range of densities we observed for yeast PM proteins and indicates a relatively homogeneous spatial distribution. Our results thus indicate that $\operatorname{Slg} 1^{\mathrm{TMD}}$ constitutes a well-suited marker to follow lateral association of TMDs in the yeast PM. Importantly, we did not observe any significant change in the network factor for Slg $1^{\text {TMD }}$ upon removal of PS. This agrees, on a qualitative level, with the outcome of the MD simulations. Removal 
A

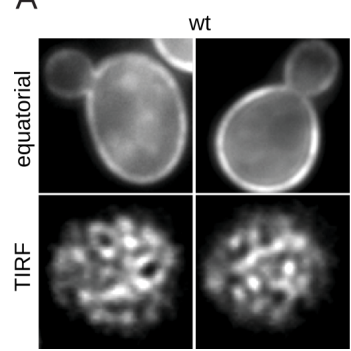

$\Delta c h o 1$

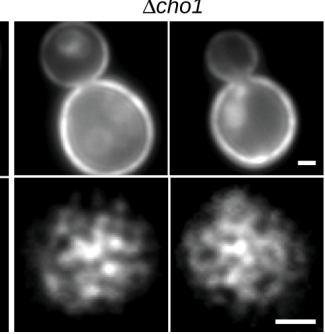

B

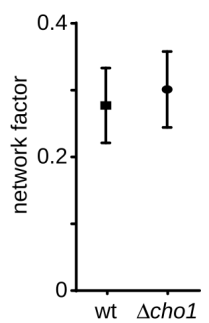

Figure 10: $M$ distribution and lipid composition. (A) Distribution of $\operatorname{Slg} 1^{\mathrm{TMD}}-\mathrm{mNeonGreen}$ in the medial section by epifluorescence (above) or in the PM by TIRFM (below) in control (wt) and phosphatidylserine-depleted ( $\Delta$ cho1) cells. Scale bars $1 \mu \mathrm{m}$. (B) PM density (net-

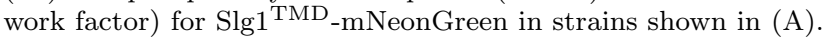

of the PS lipids changes the binding free energy by less than $0.4 \mathrm{kcal} / \mathrm{mol}$, and furthermore, has opposite signs for DOPC as compared to DIPC. Therefore, simulations would predict a very small effect with respect to the binding affinity, which is compatible with the independence of the network factor on the removal of PS.

\section{Discussion}

We have provided detailed information about the thermodynamics and kinetics of TMD dimer formation for different lipid environments. The chosen TMD Slg1 displays a significant dimerization propensity as quantified by the free energy gain $\Delta \mathrm{G}$. The convergence of PMF profiles require relatively long simulation times, consistent with a recent computational study [14], strongly suggesting the use of a coarse-grain level of description. The chosen MARTINI force field has already provided many important insights into biological processes, in particular also about the association of TMDs [13, 15, 18, 20, 56]. For the specific case of glycophorin A (GpA) results of the coarse-grain description [18] turned out to be comparable to their atomistic counterparts [57]. However, it has also been shown that atomistic simulations generally predict lower free energy values compared to coarse-grain ones and even underestimate the corresponding experimental data [58]. Another study proposed that scaling values for protein-lipid interactions in atomistic simulations would result in better agreement with experimental data and better predicts the native states, while this was not the case in coarse-grain simulations [59]. In the present work we are more interested in relative effects upon variation of the lipid composition rather than absolute free energies.

From the simulations it was possible to distinguish the pre-dimerized and dimerized states. In the pre-dimerized state, the two TMDs are only weakly coupled and represent a LH configuration, which agrees with the observations in refs. $[18,21]$. In contrast, the free energies of dimerization and the structure of the dimer depend on the lipid composition. Concerning the free energies, this dependence can be rationalized via some general characteristics. For instance, the tilt angle of the TMDs show marked differences in different systems, with DIPC giving rise to the highest tilt angle (Figure 11A). Since high tilt angles cause a reduction in the number of contacts of the $\mathrm{N}$-terminus and $\mathrm{C}$-terminus 
residues, the helix-helix association is less favorable. This correlation is somewhat stronger than with the membrane thickness as all the complex mixtures have similar thickness values (Figure 11B). Furthermore, the free energy of association is significantly correlated with the membrane order parameter, reflecting the degree of saturation: the higher the order parameter, the stronger the helixhelix association (Figure 11C). The order parameter also correlates well with the tilt angle of TMDs (Figure 11A, C).

These results agree with the outcome of a previous simulation study on GpA TMDs, where the correlation of dimer association with the order parameter was high and no correlation with membrane thickness was observed [20]. Two other aspects of that work are also worth to be compared with our results. (1). It is stated that the presence of lipids that are strongly bound to dimerized TMDs, reduce the stability of the dimer due to the loss of entropy of these lipids. Here we see that PS-lipids closely interact with the dimer due to the attraction of PS lipids to the positively-charged residues at the intracellular side of the TMDs. However, on average we do not observe a significant change of the dimer stability upon addition of PS-lipids (once slightly stabilizing, once slight destabilizing, depending on the saturation states of the acyl chains of lipids). The GpA TMD in E. coli membranes, however, showed significant destabilization in the presence of anionic lipids, rationalized by the electrostatic interactions between the membrane and the positively charged residues of the protein [25]. Considering the similarity of the presence of the positively charged residues in the C-terminus of the GpA TMD in the aforementioned study as the studied TMD here, this difference might be due to the marked interaction of ergosterol with the TMDs in our simulations and interfering with the effect of PS lipids. (2) That work reports a small repulsion of the TMDs for intermediate distances around $2.5 \mathrm{~nm}$. This is not observed in our free energy curves.

We would also like to mention a combined experimental and simulation study of bitopic proteins has shown that the free energy of dimerization is stronger in DLPC systems as compared to DPPC and POPC systems; and therefore, it was argued that neither the order parameter nor membrane thickness is correlated with dimer stability [60]. These different observations show that a comparison between different proteins and lipid compositions have to be performed with care. Therefore, it would be interesting to incorporate different TMDs in the complex mixtures of lipids in order to understand the relevance of the proteins in lipid-protein interactions. This has been already performed in model membranes with only one lipid type and different TMDs have been shown to even exhibit even opposed behavior [61]. The impact of cholesterol on dimer formation has already been studied for various systems [8-10, 14, 61]. It was also shown that ergosterol interacts with the GxxxG motif in the N-terminal part of TMDs, similar to cholesterol $[11,13,15,62,63]$. This strong interaction of ergosterol with TMDs may also be responsible for some of the characteristics of dimer formation, including the crossing angle state. Indeed, we saw that ergosterol interacts differently with the dimer in the POPC-Erg and DOPC systems as compared to the DIPC systems, and is a major contributor to the difference in interaction between saturated and unsaturated lipids with TMD residues (Figure 8). Its higher tilt in DIPC systems increases the interaction with the residues located at the hydrophobic core of the membrane, whereas the interaction is reduced with the $\mathrm{N}$ - and $\mathrm{C}$-terminal residues.

Of key relevance is also the presence of two different crossing angle states 
in dimers: $\mathrm{RH}$ and LH. Both states have been characterized in different experiments on ErB growth factors receptors [4, 64-66], or EphA2 [67], which are often ascribed to different dimer activity states imposed by altered membrane environment, and have also been observed in computational works [21, 68]. Our results also highlight the significance of membrane environment to induce different dimer states, e.g., the restriction to RH states for DIPC systems, likely responsible for various protein patterns in yeast PM. The molecular mechanism for this different behavior still needs to be revealed. The modulation of the structure of dimers by different membrane environments, comprised of only one lipid type, has also been reported for ErbB2 TMDs [24]. Interestingly, we also showed that the free energy of dimerization for POPC-Erg and DOPC systems without PS is slightly lower for LH configurations as compared to RH ones, whereas for DOPC systems with PS the opposite holds. Of course, the resulting free energy for dimer formation has to involve an appropriate average over both the $\mathrm{LH}$ and the RH state.

Furthermore, the thermodynamic free-energies estimates probabilities of LH configurations were shown to be systematically smaller than the results from our ensemble simulations. Note that the ensemble simulations can only be compared to the equilibrium case after several transitions between the $\mathrm{LH}$ and the $\mathrm{RH}$ state, which would be far beyond MD time scales. The observed preference of LH configurations in the ensemble simulations thus suggest that it is more favorable to enter the LH configuration due to properties of the barrier region. This is a natural assumption since the LH configurations dominate the predimerized state. To describe the complete energetic landscape of dimerization, including the structure of the dimer and saddle points of pre-dimerized states, a 2D PMF would be required, as was recently demonstrated using Metadynamics enhanced sampling technique $[14,21]$.

Interestingly, not only does the lipid environment affect TMD dimer stability, but dimerization can in turn influence the properties of the surrounding membrane [55]. We observed an increase in the order parameter of lipids at a distance of around $1 \mathrm{~nm}$ from the dimer, whereas no specific change was detected for monomers. The ordering effect was stronger for membranes with already higher order parameter. This is in agreement with stronger variation of membrane thickness around ErbB2 dimers in DPPC as compared to DLPC bilayers [24]. We speculate that this represents a self-reinforcing mechanism for dimer formation, since the dimerization is more preferable for systems with higher order. A similar behavior has been reported in a previous study, where dimerization of Bnip3 TMDs led to an increased order parameter in a POPC membrane [61]. Interestingly, for DIPC systems the lipid-protein interaction starts at a considerably larger distance than for DOPC systems, which was also reflected in the variation of the tilt angle of ergosterol with respect to the position of the dimer. Therefore, a higher degree of unsaturation in the acyl chains induces long-range perturbation in the membrane. Naturally, the change of order around a dimer may affect the mutual interaction of TMD dimers, possibly giving rise to larger clusters of TMDs.

Our approach suggests a possible connection between the patchwork patterns of TMDs in the yeast PM and the free energy calculations in our MD simulations. In both approaches we have examined realistic yeast PM compositions, whereas in NMR experiments in order to estimate the free energy of TMD interactions usually mimetic membranes are used [23, 69-71]. Interestingly, despite the 

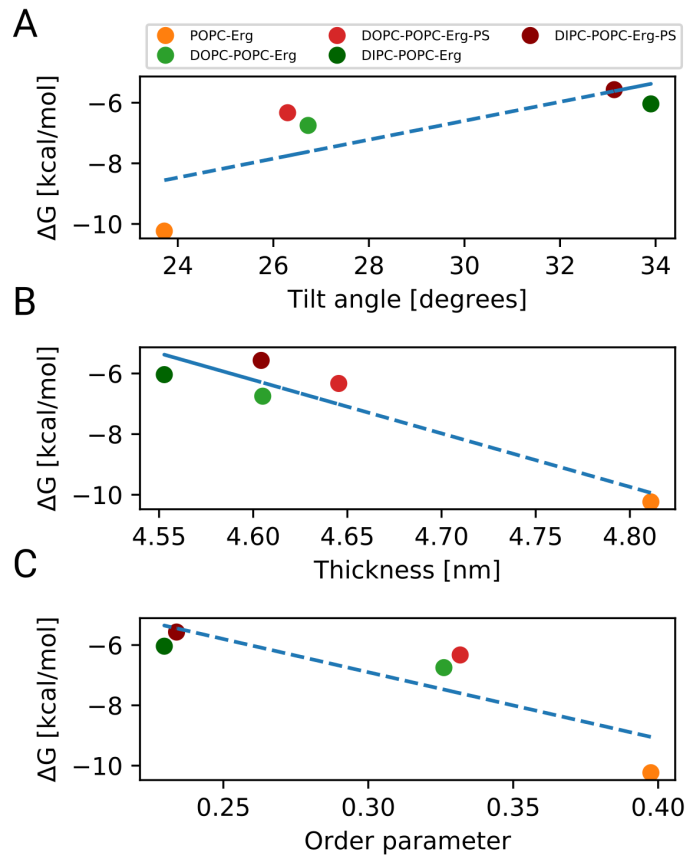

Figure 11: $\Delta \mathrm{G}$ as a function of (A) tilt angle, (B) membrane thickness and (C) PLs order parameter.

wide-spread effects of PS depletion on pattern formation in the yeast PM [2], both MD simulations and the live cell TIRF images indicated that PS lipids do not significantly influence association of the $\mathrm{Slg}_{\mathrm{TMD}}$. Future experiments will determine whether lipid saturation or other lipid varieties exert direct influence on Slg1 clustering. Furthermore, future simulations on TMDs, having shown experimental PS-dependencies in [2], may help to reveal under which conditions a PS-dependency on the dimer formation may be observed.

\section{Conclusions}

We have studied the interaction of TMDs in a biologically relevant yeast PM, combining microscopic coarse-grain MD simulations with high-resolution microscopy experiments. Whereas the MD simulations allow a detailed characterization of local structural and thermodynamic properties, the experiments reveal aspects of the resulting structures on the $100 \mathrm{~nm}$ scale and beyond. We have shown that non-specific protein-lipid interactions can have significant effects on the conformation and stability of resulting dimers as well as on the free energy of dimerization. In particular, the level of acyl chain saturation was found to have a strong impact on TMD association. Importantly, the membrane environment is modulated upon TMD dimerization, as measured by changes in the phospholipid order parameter and the orientation of ergosterol. Dimerization in turn leads to long-range perturbations in the surrounding lipids, which penetrate further in the presence of unsaturated lipids. Our results highlight again 
the complex mutual interplay between the TMD and lipid environment. The role of anionic PS lipids turned out to be negligible, both observed via the free energy of association in MD simulations, and via the network factor in protein patterns in the yeast PM. Ultimately, our integrated approach aims to bridge the different scales of analysis and improve our understanding of lipid-TMD interaction during organization of biological membranes.

\section{Author Contributions}

AA, RWS, and AH conceived and designed the analysis. AA performed the computer simulations and the subsequent analysis. AE performed the experiments and the related analysis. All authors have contributed to the final version of manuscript.

\section{Acknowledgment}

We acknowledge initial discussions with Fabian Keller, and the financial support by the German Science Foundation (DFG) via SFB 1348 (to AH and RWS) and SFB 944 (to RWS). 


\section{References}

[1] Christian Schuberth and Roland Wedlich-Söldner. Building a patchwork The yeast plasma membrane as model to study lateral domain formation. Biochimica et Biophysica Acta (BBA) - Molecular Cell Research, 1853(4):767-774, 2015. ISSN 01674889. doi: https://doi.org/10.1016/j.bbamcr.2014.12.019. URL https://www.sciencedirect.com/science/article/pii/S0167488914004479.

[2] Felix Spira, Nikola S. Mueller, Gisela Beck, Philipp Von Olshausen, Joachim Beig, and Roland Wedlich-Söldner. Patchwork organization of the yeast plasma membrane into numerous coexisting domains. Nature Cell Biology, 14(6):640-648, 2012. ISSN 14657392. doi: 10.1038/ncb2487. URL http://dx.doi .org/10.1038/ncb2487.

[3] Nikola S Mueller, Roland Wedlich-Söldner, and Felix Spira. From mosaic to patchwork: Matching lipids and proteins in membrane organization. Molecular Membrane Biology, 29(5):186-196, aug 2012. ISSN 0968-7688. doi: 10.3109/09687688.2012.687461. URL https ://doi.org/10.3109/09687688.2012.687461.

[4] Eduard V. Bocharov, Konstantin S. Mineev, Konstantin V. Pavlov, Sergey A. Akimov, Andrey S. Kuznetsov, Roman G. Efremov, and Alexander S. Arseniev. Helix-helix interactions in membrane domains of bitopic proteins: Specificity and role of lipid environment. Biochimica et Biophysica Acta - Biomembranes, 1859(4):561576, 2017. ISSN 18792642. doi: 10.1016/j.bbamem.2016.10.024. URL http://dx.doi.org/10.1016/j.bbamem.2016.10.024.

[5] R F S Walters and W F DeGrado. Helix-packing motifs in membrane proteins. Proceedings of the National Academy of Sciences, 103 (37):13658 LP - 13663, sep 2006. doi: 10.1073/pnas.0605878103. URL http: //www . pnas.org/content/103/37/13658. abstract.

[6] Shao-Qing Zhang, Daniel W. Kulp, Chaim A. Schramm, Marco Mravic, Ilan Samish, and William F. DeGrado. The Membraneand Soluble-Protein Helix-Helix Interactome: Similar Geometry via Different Interactions. Structure, 23(3):527-541, 2015. ISSN 0969-2126. doi: https://doi.org/10.1016/j.str.2015.01.009. URL http://www.sciencedirect.com/science/article/pii/S0969212615000337.

[7] Alessandro Senes, Mark Gerstein, and Donald M Engelman. Statistical analysis of amino acid patterns in transmembrane helices: the GxxxG motif occurs frequently and in association with $\beta$ branched residues at neighboring positions11Edited by G. von Heijne. Journal of Molecular Biology, 296(3):921-936, 2000. ISSN 0022-2836. doi: https://doi.org/10.1006/jmbi.1999.3488. URL https://www.sciencedirect.com/science/article/pii/S0022283699934885.

[8] Veerappan Anbazhagan and Dirk Schneider. The membrane environment modulates self-association of the human GpA TM domainImplications for membrane protein folding and transmembrane signaling. Biochimica 
et Biophysica Acta (BBA) - Biomembranes, 1798(10):1899-1907, 2010. ISSN 0005-2736. doi: https://doi.org/10.1016/j.bbamem.2010.06.027. URL http://www.sciencedirect.com/science/article/pii/S0005273610002257.

[9] Sanjay Mall, Robert Broadbridge, Ram. P. Sharma, J. Malcolm East, and Anthony G. Lee. Self-Association of Model Transmembrane $\alpha$-Helices Is Modulated by Lipid Structure. Biochemistry, 40(41):12379-12386, sep 2001. doi: 10.1021/bi011075y.

[10] Lidia Cristian, James D Lear, and William F DeGrado. Use of thiol-disulfide equilibria to measure the energetics of assembly of transmembrane helices in phospholipid bilayers. Proceedings of the National Academy of Sciences, 100(25):14772 LP - 14777, dec 2003. doi: 10.1073/pnas.2536751100. URL http: //www . pnas.org/content/100/25/14772 . abstract.

[11] Julie Grouleff, Sheeba Jem Irudayam, Katrine K Skeby, and Birgit Schiøtt. The influence of cholesterol on membrane protein structure, function, and dynamics studied by molecular dynamics simulations. Biochimica et Biophysica Acta (BBA) - Biomembranes, 1848(9):1783-1795, 2015. ISSN 0005-2736. doi: https://doi.org/10.1016/j.bbamem.2015.03.029. URL http://www.sciencedirect.com/science/article/pii/S000527361500108X.

[12] Muhammad Hasan, Dharmesh Patel, Natalie Ellis, Steven P Brown, Józef R Lewandowski, and Ann M Dixon. Modulation of Transmembrane Domain Interactions in Neu Receptor Tyrosine Kinase by Membrane Fluidity and Cholesterol. The Journal of Membrane Biology, 252(4): 357-369, 2019. ISSN 1432-1424. doi: 10.1007/s00232-019-00075-4. URL https://doi.org/10.1007/s00232-019-00075-4.

[13] Anupam Prakash, Lorant Janosi, and Manolis Doxastakis. GxxxG Motifs, Phenylalanine, and Cholesterol Guide the Self-Association of Transmembrane Domains of ErbB2 Receptors. Biophysical Journal, 101(8):1949 1958, oct 2011. ISSN 0006-3495. doi: 10.1016/j.bpj.2011.09.017. URL https://doi.org/10.1016/j.bpj.2011.09.017.

[14] Aiswarya B Pawar and Durba Sengupta. Role of Cholesterol in Transmembrane Dimerization of the ErbB2 Growth Factor Receptor. The Journal of Membrane Biology, 2021. ISSN 1432-1424. doi: 10.1007/s00232-021-00168z. URL https://doi.org/10.1007/s00232-021-00168-z.

[15] Nadine Flinner and Enrico Schleiff. Dynamics of the Glycophorin A Dimer in Membranes of Native-Like Composition Uncovered by Coarse-Grained Molecular Dynamics Simulations. PLOS ONE, 10(7):e0133999, jul 2015. URL https://doi.org/10.1371/journal.pone.0133999.

[16] Mar Orzáez, Dunja Lukovic, Concepción Abad, Enrique Pérez-Payá, and Ismael Mingarro. Influence of hydrophobic matching on association of model transmembrane fragments containing a minimised glycophorin A dimerisation motif. FEBS Letters, 579(7):1633-1638, 2005. ISSN 0014-5793. doi: https://doi.org/10.1016/j.febslet.2005.01.078. URL http://www.sciencedirect.com/science/article/pii/S0014579305002085. 
[17] Emma Sparr, Walter L Ash, Petr V Nazarov, Dirk T S Rijkers, Marcus A Hemminga, D Peter Tieleman, and J Antoinette Killian. Self-association of Transmembrane $\alpha$-Helices in Model Membranes: IMPORTANCE OF HELIX ORIENTATION AND ROLE OF HYDROPHOBIC MISMATCH. Journal of Biological Chemistry, 280 (47):39324-39331, nov 2005. doi: 10.1074/jbc.M502810200. URL http: //www. jbc.org/content/280/47/39324. abstract.

[18] Durba Sengupta and Siewert J Marrink. Lipid-mediated interactions tune the association of glycophorin A helix and its disruptive mutants in membranes. Physical Chemistry Chemical Physics, 12(40):1298712996, 2010. ISSN 1463-9076. doi: 10.1039/C0CP00101E. URL http://dx.doi.org/10.1039/COCP00101E.

[19] A S Kuznetsov, P E Volynsky, and R G Efremov. Role of the Lipid Environment in the Dimerization of Transmembrane Domains of Glycophorin A. Acta naturae, 7(4):122-127, 2015. ISSN 2075-8251. URL https://pubmed.ncbi.nlm.nih.gov/26798499 https://www.ncbi.nlm.nih.gov/pmc/articles/PMC4717257/.

[20] Lorant Janosi, Anupam Prakash, and Manolis Doxastakis. Lipid-modulated sequence-specific association of glycophorin A in membranes. Biophysical journal, 99 1:284-292, 2010.

[21] Mickaël Lelimousin, Vittorio Limongelli, and Mark S. P. Sansom. Conformational Changes in the Epidermal Growth Factor Receptor: Role of the Transmembrane Domain Investigated by Coarse-Grained MetaDynamics Free Energy Calculations. Journal of the American Chemical Society, 138 (33):10611-10622, aug 2016. doi: 10.1021/jacs.6b05602.

[22] Luis Martínez-Gil Brayan Grau, Matti Javanainen, Maria Jesús GarcíaMurria, Waldemar Kulig, Ilpo Vattulainen, Ismael Mingarro. The role of hydrophobic matching on transmembrane helix packing in cells. Cell Stress, 1(2):90-106, 2017. doi: 10.15698/cst2017.11.111.

[23] Pavel E Bragin, Konstantin S Mineev, Olga V Bocharova, Pavel E Volynsky, Eduard V Bocharov, and Alexander S Arseniev. HER2 Transmembrane Domain Dimerization Coupled with Self-Association of Membrane-Embedded Cytoplasmic Juxtamembrane Regions. Journal of Molecular Biology, 428(1):52-61, 2016. ISSN 00222836. doi: https://doi.org/10.1016/j.jmb.2015.11.007. URL https://www.sciencedirect.com/science/article/pii/S0022283615006506.

[24] Srinivasa M Gopal, Aiswarya B Pawar, Tsjerk A Wassenaar, and Durba Sengupta. Lipid-dependent conformational landscape of the ErbB2 growth factor receptor dimers. Chemistry and Physics of Lipids, 230:104911, 2020. ISSN 0009-3084. doi: https://doi.org/10.1016/j.chemphyslip.2020.104911. URL https://www.sciencedirect.com/science/article/pii/S0009308420300426.

[25] Heedeok Hong and James U. Bowie. Dramatic Destabilization of Transmembrane Helix Interactions by Features of Natural Membrane Environments. Journal of the American Chemical Society, 133(29):11389-11398, jul 2011. doi: 10.1021/ja204524c. 
[26] George Hedger, David Shorthouse, Heidi Koldsø, and Mark S. P. Sansom. Free Energy Landscape of Lipid Interactions with Regulatory Binding Sites on the Transmembrane Domain of the EGF Receptor. The Journal of Physical Chemistry B, 120(33):8154-8163, may 2016. doi: 10.1021/acs.jpcb.6b01387.

[27] Jan Domański, George Hedger, Robert B. Best, Phillip J. Stansfeld, and Mark S. P. Sansom. Convergence and Sampling in Determining Free Energy Landscapes for Membrane Protein Association. The Journal of Physical Chemistry B, 121(15):3364-3375, nov 2016. doi: 10.1021/acs.jpcb.6b08445.

[28] Andrey S. Kuznetsov, Anton A. Polyansky, Markus Fleck, Pavel E. Volynsky, and Roman G. Efremov. Adaptable Lipid Matrix Promotes ProteinProtein Association in Membranes. Journal of Chemical Theory and Computation, 11(9):4415-4426, aug 2015. doi: 10.1021/acs.jctc.5b00206.

[29] Xavier Prasanna, P J Praveen, and Durba Sengupta. Sequence dependent lipid-mediated effects modulate the dimerization of ErbB2 and its associative mutants. Physical Chemistry Chemical Physics, 15(43): 19031-19041, 2013. ISSN 1463-9076. doi: 10.1039/C3CP52447G. URL http://dx.doi.org/10.1039/C3CP52447G.

[30] M A Lemmon, J M Flanagan, J F Hunt, B D Adair, B J Bormann, C E Dempsey, and D M Engelman. Glycophorin A dimerization is driven by specific interactions between transmembrane alpha-helices. Journal of Biological Chemistry, 267(11):7683-7689, 1992. ISSN 0021-9258. doi: https://doi.org/10.1016/S0021-9258(18)42569-0. URL https://www.sciencedirect.com/science/article/pii/S0021925818425690.

[31] Kevin R MacKenzie, James H Prestegard, and Donald M Engelman. A Transmembrane Helix Dimer: Structure and Implications. Science, 276 (5309):131 LP - 133, apr 1997. doi: 10.1126/science.276.5309.131. URL http://science.sciencemag.org/content/276/5309/131.abstract.

[32] Sanguk Kim, Tae-Joon Jeon, Amit Oberai, Duan Yang, Jacob J Schmidt, and James U Bowie. Transmembrane glycine zippers: Physiological and pathological roles in membrane proteins. Proceedings of the $\mathrm{Na}$ tional Academy of Sciences of the United States of America, 102(40): 14278 LP - 14283, oct 2005. doi: 10.1073/pnas.0501234102. URL http: //www .pnas.org/content/102/40/14278. abstract.

[33] Jon V Busto, Annegret Elting, Daniel Haase, Felix Spira, Julian Kuhlman, Marco Schäfer-Herte, and Roland Wedlich-Söldner. Lateral plasma membrane compartmentalization links protein function and turnover. The EMBO journal, 37(16):e99473, aug 2018. ISSN 1460-2075. doi: 10.15252/embj.201899473. URL https://pubmed.ncbi.nlm.nih.gov/29976762 https://www.ncbi.nlm.nih.gov/pmc/articles/PMC6092676/.

[34] E Zinser, C D Sperka-Gottlieb, E V Fasch, S D Kohlwein, F Paltauf, and G Daum. Phospholipid synthesis and lipid composition of subcellular membranes in the unicellular eukaryote 
Saccharomyces cerevisiae. Journal of bacteriology, 173(6):20262034, mar 1991. ISSN 0021-9193. doi: 10.1128/jb.173.6.20262034.1991. URL https://pubmed.ncbi.nlm.nih.gov/2002005 https://www.ncbi.nlm.nih.gov/pmc/articles/PMC207737/.

[35] E Zinser, F Paltauf, and G Daum. Sterol composition of yeast organelle membranes and subcellular distribution of enzymes involved in sterol metabolism. Journal of bacteriology, 175(10):28532858, may 1993. ISSN 0021-9193. doi: 10.1128/jb.175.10.28532858.1993. URL https://pubmed.ncbi.nlm.nih.gov/8491706 https://www.ncbi.nlm.nih.gov/pmc/articles/PMC204601/.

[36] Gerrit van Meer, Dennis R Voelker, and Gerald W Feigenson. Membrane lipids: where they are and how they behave. Nature Reviews Molecular Cell Biology, 9(2):112-124, 2008. ISSN 1471-0080. doi: 10.1038/nrm2330. URL https://doi.org/10.1038/nrm2330.

[37] J H Lorent, K R Levental, L Ganesan, G Rivera-Longsworth, E Sezgin, M Doktorova, E Lyman, and I Levental. Plasma membranes are asymmetric in lipid unsaturation, packing and protein shape. Nature Chemical Biology, 16(6):644-652, 2020. ISSN 1552-4469. doi: 10.1038/s41589-0200529-6. URL https://doi .org/10.1038/s41589-020-0529-6.

[38] Andrew Waterhouse, Martino Bertoni, Stefan Bienert, Gabriel Studer, Gerardo Tauriello, Rafal Gumienny, Florian T Heer, Tjaart A P de Beer, Christine Rempfer, Lorenza Bordoli, Rosalba Lepore, and Torsten Schwede. SWISS-MODEL: homology modelling of protein structures and complexes. Nucleic acids research, 46(W1): W296-W303, jul 2018. ISSN 1362-4962. doi: 10.1093/nar/gky427. URL https://pubmed.ncbi.nlm.nih.gov/29788355 https://www.ncbi.nlm.nih.gov/pmc/articles/PMC6030848/.

[39] Sunhwan Jo, Taehoon Kim, Vidyashankara G Iyer, and Wonpil Im. CHARMM-GUI: A web-based graphical user interface for CHARMM. Journal of Computational Chemistry, 29(11):18591865, 2008. ISSN 1096-987X. doi: 10.1002/jcc.20945. URL http://dx.doi.org/10.1002/jcc. 20945.

[40] Siewert J. Marrink, Alex H. de Vries, and Alan E. Mark. Coarse Grained Model for Semiquantitative Lipid Simulations. The Journal of Physical Chemistry B, 108(2):750-760, jan 2004. ISSN 1520-6106. doi: 10.1021/jp036508g. URL http://pubs . acs .org/doi/abs/10.1021/jp036508g.

[41] Djurre H. de Jong, Gurpreet Singh, W. F. Drew Bennett, Clement Arnarez, Tsjerk A. Wassenaar, Lars V. Schäfer, Xavier Periole, D. Peter Tieleman, and Siewert J. Marrink. Improved Parameters for the Martini CoarseGrained Protein Force Field. Journal of Chemical Theory and Computation, 9(1):687-697, nov 2012. doi: 10.1021/ct300646g.

[42] Siewert J. Marrink, H. Jelger Risselada, Serge Yefimov, D. Peter Tieleman, and Alex H. De Vries. The MARTINI force field: Coarse grained model 
for biomolecular simulations. Journal of Physical Chemistry B, 111(27): 7812-7824, 2007. ISSN 15206106. doi: 10.1021/jp071097f.

[43] Erik Lindahl, Berk Hess, and David van der Spoel. GROMACS 3.0: a package for molecular simulation and trajectory analysis. Molecular modeling annual, 7(8):306-317, 2001. ISSN 0948-5023. doi: 10.1007/s008940100045. URL https://doi.org/10.1007/s008940100045.

[44] David Van Der Spoel, Erik Lindahl, Berk Hess, Gerrit Groenhof, Alan E Mark, and Herman J C Berendsen. GROMACS: Fast, flexible, and free. Journal of Computational Chemistry, 26(16):17011718, dec 2005. ISSN 0192-8651. doi: 10.1002/jcc.20291. URL https://doi.org/10.1002/jcc. 20291.

[45] H J C Berendsen, J P M Postma, W F van Gunsteren, A DiNola, and J R Haak. Molecular dynamics with coupling to an external bath. The Journal of Chemical Physics, 81(8):3684-3690, oct 1984. ISSN 0021-9606. doi: 10.1063/1.448118. URL https ://doi.org/10.1063/1.448118.

[46] M Parrinello and A Rahman. Polymorphic transitions in single crystals: A new molecular dynamics method. Journal of Applied Physics, 52(12): 7182-7190, dec 1981. ISSN 0021-8979. doi: 10.1063/1.328693. URL https://doi.org/10.1063/1.328693.

[47] Naveen Michaud-Agrawal, Elizabeth J Denning, Thomas B Woolf, and Oliver Beckstein. MDAnalysis: A Toolkit for the Analysis of Molecular Dynamics Simulations. Journal of computational chemistry, 32(10): 2319-2327, jul 2011. ISSN 0192-8651. doi: 10.1002/jcc.21787. URL http://www.ncbi.nlm.nih.gov/pmc/articles/PMC3144279/.

[48] Richard J Gowers, Max Linke, Jonathan Barnoud, Tyler J E Reddy, Manuel N Melo, Sean L Seyler, David L Dotson, Sébastien Buchoux, Ian M Kenney, and Oliver Beckstein. MDAnalysis : A Python Package for the Rapid Analysis of Molecular Dynamics Simulations MDAnalysis. (Scipy): 98-105, 2016.

[49] William Humphrey, Andrew Dalke, and Klaus Schulten. VMD: Visual molecular dynamics. Journal of Molecular Graphics, 14(1):33-38, 1996. ISSN 0263-7855. doi: http://dx.doi.org/10.1016/0263-7855(96)00018-5. URL http://www.sciencedirect.com/science/article/pii/0263785596000185.

[50] Cyrus Chothia, Michael Levitt, and Douglas Richardson. Helix to helix packing in proteins. Journal of Molecular Biology, 145(1):215-250, 1981. ISSN 0022-2836. doi: https://doi.org/10.1016/0022-2836(81)90341-7. URL http://www.sciencedirect.com/science/article/pii/0022283681903417.

[51] G M Torrie and J P Valleau. Nonphysical sampling distributions in Monte Carlo free-energy estimation: Umbrella sampling. Journal of Computational Physics, 23(2):187-199, 1977. ISSN 00219991. doi: https://doi.org/10.1016/0021-9991(77)90121-8. URL https://www.sciencedirect.com/science/article/pii/0021999177901218. 
[52] Shankar Kumar, John Rosenberg, Djamal Bouzida, and Robert Swendsen. The weighted histogram analysis method for free-energy calculations on biomolecules, volume 13. oct 1992. doi: 10.1002/jcc.540130812.

[53] Tomasz Róg, Marta Pasenkiewicz-Gierula, Ilpo Vattulainen, and Mikko Karttunen. Ordering effects of cholesterol and its analogues, jan 2009. ISSN 00052736. URL https://www.sciencedirect.com/science/article/pii/S0005273608002721.

[54] Maurits R. R. de Planque and J. Antoinette Killian*. Proteinlipid interactions studied with designed transmembrane peptides: role of hydrophobic matching and interfacial anchoring (Review). Molecular Membrane Biology, 20(4):271-284, oct 2003. ISSN 0968-7688. doi: 10.1080/09687680310001605352. URL http: //www.tandfonline.com/doi/full/10.1080/09687680310001605352.

[55] Morten Ø Jensen and Ole G Mouritsen. Lipids do influence protein functionthe hydrophobic matching hypothesis revisited. Biochimica et Biophysica Acta (BBA) - Biomembranes, 1666(1):205-226, 2004. ISSN 0005-2736. doi: https://doi.org/10.1016/j.bbamem.2004.06.009. URL https://www.sciencedirect.com/science/article/pii/S0005273604001634.

[56] Stefan Gahbauer and Rainer A. Böckmann. Comprehensive Characterization of Lipid-Guided G Protein-Coupled Receptor Dimerization. The journal of physical chemistry. B, 124(14):2823-2834, apr 2020. ISSN 15205207. doi: $10.1021 /$ acs.jpcb.0c00062.

[57] Jérôme Hénin, Andrew Pohorille, and Christophe Chipot. Insights into the Recognition and Association of Transmembrane $\alpha$-Helices. The Free Energy of $\alpha$-Helix Dimerization in Glycophorin A. Journal of the American Chemical Society, 127(23):8478-8484, may 2005. doi: 10.1021/ja050581y.

[58] Manami Nishizawa and Kazuhisa Nishizawa. Potential of mean force analysis of the self-association of leucine-rich transmembrane $\alpha$-helices: Difference between atomistic and coarse-grained simulations. The Journal of Chemical Physics, 141(7):75101, aug 2014. ISSN 0021-9606. doi: 10.1063/1.4891932. URL https://doi.org/10.1063/1.4891932.

[59] Jan Domański, Mark S. P. Sansom, Phillip J. Stansfeld, and Robert B. Best. Balancing Force Field ProteinLipid Interactions To Capture Transmembrane HelixHelix Association. Journal of Chemical Theory and Computation, 14(3):1706-1715, feb 2018. doi: 10.1021/acs.jctc.7b00983.

[60] P E Bragin, A S Kuznetsov, O V Bocharova, P E Volynsky, A S Arseniev, R G Efremov, and K S Mineev. Probing the effect of membrane contents on transmembrane protein-protein interaction using solution NMR and computer simulations. Biochimica et Biophysica Acta (BBA) - Biomembranes, 1860(12):2486-2498, 2018. ISSN 0005-2736. doi: https://doi.org/10.1016/j.bbamem.2018.09.013. URL http://www.sciencedirect.com/science/article/pii/S0005273618302773.

[61] Anton A. Polyansky, Pavel E. Volynsky, and Roman G. Efremov. Multistate Organization of Transmembrane Helical Protein Dimers Governed by 
the Host Membrane. Journal of the American Chemical Society, 134(35): 14390-14400, aug 2012. doi: 10.1021/ja303483k.

[62] Frédérick J-M de Meyer, Jocelyn M Rodgers, Thomas F Willems, and Berend Smit. Molecular simulation of the effect of cholesterol on lipid-mediated protein-protein interactions. Biophysical journal, 99(11): 3629-3638, dec 2010. ISSN 1542-0086. doi: 10.1016/j.bpj.2010.09.030. URL https://pubmed.ncbi.nlm.nih.gov/21112287 https://www.ncbi.nlm.nih.gov/pmc/articles/PMC2998597/.

[63] Paul J Barrett, Yuanli Song, Wade D Van Horn, Eric J Hustedt, Johanna M Schafer, Arina Hadziselimovic, Andrew J Beel, and Charles R Sanders. The Amyloid Precursor Protein Has a Flexible Transmembrane Domain and Binds Cholesterol. Science, 336(6085): 1168 LP - 1171, jun 2012. doi: 10.1126/science.1219988. URL http://science.sciencemag.org/content/336/6085/1168. abstract.

[64] Eduard V Bocharov, Konstantin S Mineev, Pavel E Volynsky, Yaroslav S Ermolyuk, Elena N Tkach, Alexander G Sobol, Vladimir V Chupin, Michail P Kirpichnikov, Roman G Efremov, and Alexander S Arseniev. Spatial Structure of the Dimeric Transmembrane Domain of the Growth Factor Receptor ErbB2 Presumably Corresponding to the Receptor Active State*. Journal of Biological Chemistry, 283(11):6950-6956, 2008. ISSN 0021-9258. doi: https://doi.org/10.1074/jbc.M709202200. URL https://www.sciencedirect.com/science/article/pii/S0021925820571330.

[65] K S Mineev, N F Khabibullina, E N Lyukmanova, D A Dolgikh, M P Kirpichnikov, and A S Arseniev. Spatial structure and dimermonomer equilibrium of the ErbB3 transmembrane domain in DPC micelles. Biochimica et Biophysica Acta (BBA) - Biomembranes, 1808(8):2081-2088, 2011. ISSN 0005-2736. doi: https://doi.org/10.1016/j.bbamem.2011.04.017. URL https://www.sciencedirect.com/science/article/pii/S0005273611001337.

[66] Aiswarya B Pawar and Durba Sengupta. Resolving the conformational dynamics of ErbB growth factor receptor dimers. Journal of Structural Biology, 207(2):225-233, 2019. ISSN 10478477. doi: https://doi.org/10.1016/j.jsb.2019.05.013. URL https://www.sciencedirect.com/science/article/pii/S1047847719301297.

[67] Eduard V Bocharov, Maxim L Mayzel, Pavel E Volynsky, Konstantin S Mineev, Elena N Tkach, Yaroslav S Ermolyuk, Alexey A Schulga, Roman G Efremov, and Alexander S Arseniev. Left-Handed Dimer of EphA2 Transmembrane Domain: Helix Packing Diversity among Receptor Tyrosine Kinases. Biophysical Journal, 98(5):881-889, 2010. ISSN 0006-3495. doi: https://doi.org/10.1016/j.bpj.2009.11.008. URL http://www.sciencedirect.com/science/article/pii/S0006349509017329.

[68] Benjamin A. Hall, Khairul Bariyyah Abd Halim, Amanda Buyan, Beatrice Emmanouil, and Mark S. P. Sansom. Sidekick for Membrane Simulations: Automated Ensemble Molecular Dynamics Simulations of Transmembrane Helices. Journal of Chemical Theory and Computation, 10(5):2165-2175, apr 2014. doi: 10.1021/ct500003g. 
[69] Konstantin S Mineev, Dmitry M Lesovoy, Dinara R Usmanova, Sergey A Goncharuk, Mikhail A Shulepko, Ekaterina N Lyukmanova, Mikhail P Kirpichnikov, Eduard V Bocharov, and Alexander $\mathrm{S}$ Arseniev. NMR-based approach to measure the free energy of transmembrane helixhelix interactions. Biochimica et Biophysica Acta (BBA) - Biomembranes, 1838(1, Part B):164-172, 2014. ISSN 0005-2736. doi: https://doi.org/10.1016/j.bbamem.2013.08.021. URL https://www.sciencedirect.com/science/article/pii/S0005273613003003.

[70] Konstantin S. Mineev, Stanislava V. Panova, Olga V. Bocharova, Eduard V. Bocharov, and Alexander S. Arseniev. The Membrane Mimetic Affects the Spatial Structure and Mobility of EGFR Transmembrane and Juxtamembrane Domains. Biochemistry, 54(41):6295-6298, oct 2015. doi: 10.1021/acs.biochem.5b00851.

[71] Eduard V Bocharov, Konstantin S Mineev, Marina V Goncharuk, and Alexander S Arseniev. Structural and thermodynamic insight into the process of weak dimerization of the ErbB4 transmembrane domain by solution NMR. Biochimica et Biophysica Acta (BBA) - Biomembranes, 1818(9):2158-2170, 2012. ISSN 00052736. doi: https://doi.org/10.1016/j.bbamem.2012.05.001. URL http://www.sciencedirect.com/science/article/pii/S0005273612001496. 